\title{
DELIMITACIÓN DE «LO QUE EL ALUMNO SABE» A PARTIR DE OBJETIVOS Y MODELOS DE ENSEÑANZA
}

\author{
MARÍN, N., ${ }^{1}$ JIMÉNEZ GÓMEZ, E. ${ }^{2}$ y BENARROCH, $A .^{3}$ \\ ' Departamento de Didáctica. Facultad de Ciencias de la Educación. Universidad de Almería. \\ ${ }^{2}$ Departamento de Didáctica de las Ciencias Experimentales. Facultad de Educación. \\ Universidad de Murcia. \\ ${ }^{3}$ Departamento de Didáctica de las Ciencias Experimentales. Escuela de Magisterio de Melilla. \\ Universidad de Granada.
}

\section{SUMMARY}

The educational principle proposed by Ausubel: «to find out the knowledge of the pupil and teach him consequently» is assumed by most of the educators; however, this principle does not delimit the sentence: «the knowledge of the pupit». So this sentence can have different interpretations according to the information the teacher requests from the pupil's cognitive structure to carry out the instructional process that, at the same time, depends on the teaching objetives. From here, in this paper we attempt to delimit «the knowledge of the pupil» about science as useful information to carry out the design of teaching based on the Altenative Conception Movement, Ausubel's Theory and Piaget's Theory.

\section{INTRODUCCION}

En las últimas décadas sc ha asistido, en el dominio de la didáctica de las ciencias, a una progresiva convergencia para desarroliar el trabajo educativo y de investigación bajo la concepción constructivista del aprendizaje, to que algunos autores han denominado movimiento de las concepcioncs alternativas (MCA, véase, por ejemplo, Gilbert y Swift, 1985). Desde este punto de vista, los alumnos construyen los nuevos conocimientos a partir de sus ideas o concepciones previas, de forma que la enseñanza de la ciencia consistiría fundamentalmente en promover el cambio de dichas ideas con el fin de acercarlus progresivamente al entramado conceptual académicamente aceptado.

Sin embargo, una revisión breve de la historia de la đidáctica de las ciencias hace ver, en nuestra opinión, que ha habido dos formas diferentes de conceptuatizar el estatus cognoscitivo inicial del alumno, dando lugar a opciones didácticas y curriculares bien diferentes.

Por un Jado, en la década de los sesenta y setenta, con el apogco de la teoría piagetiana para fundamentar la investigación en enseñanza de las ciencias (Gómez et al., 1992), se utilizaba el nivel cognoscitivo de 1 alumno como la variable más importante sobre la que había que fundamentar la selección y secuenciación de los conteridos de enseñanza (Shayer y Adey, 1984).

A comienzos de los ochenta, toma una gran importancia lo que sabe el alumno sobre el contenido que se le va a enseñar, ya que se pone de relieve que dichas ideas previas juegan un papel activo y decisivo en la adquisición de nuevos conocimientos (Driver, 1986). Consecuentemente se realizan numcrosos trabajos de investi- 
- Por recepción (enseñanza expositiva), donde el nuevo contenido interacciona en la memoria de trabajo (Mathieu y Thomas, 1985) con el modelo que tiene el sujeto, previo a la intervención educativa, y que alcanza significación cuanđo el conteniđo enseñado se ligue a los conceptos que el sujeto utiliza como inclusores que, a su ve $z$, sufren reconciliación integradora en la memoria a largo plazo.

Por descubrimiento, por ejemplo, mediante la resolución de problemas; sin embargo, aquí Novak y Gowin (1988, p. 20), defensores de la teoría de Ausubel, anteponen la creatividad a los modelos de la memoria temporal y a la capacidad procedimental (elementos de la memoria a largo plazo, Mathicu y Thomas, 1985, p. 241) que utilizan los sujetos a la hora de resolver una tarea.

Se podría afirmar que la propuesta de Ausubel está más referida al aprendizaje de conceptos o conocimientos declarativos (Herron, 1978; Lawson, 1983; Aliberas et ai., 1989) que al de procesos.

Si la pretensión de la enseñanza es llegar a la posibilidad de que el sujeto utilice ese nuevo contenido como una parte más de su bagaje cognoscitivo en sus conductas intelectivas ante el medio, la propuesta de Ausubel podría ser insuficiente por varios motivos:

- Es un hecho el que se puede comprender una información verbal y al poco tiempo olvidarla, pero si se adquiere por asimilación y acomodación, es decir, si el nuevo conocimiento se integra en un esquema de la estructura cognoscitiva, entonces la probabilidad de olvido es semejante a la de cualquier jucgo que se aprendió en la infancia (trompo, tabas, canicas, etc.).

- Desde la psicología del aprendizaje parece haber unanimidad en que este acto de comprensión no implica por sí solo una adquisición duradera (Beltrán, 1993).

- El conocimiento térico de un sujeto no se refiere sólo al conjunto organizado de conceptos; también lo constituyen las estrategias y reglas que permiten la valoración individual de los hechos y acontecimientos, así como los procedimientos que permiten utilizar y justificar este conocimiento (Duschl y Gitomer, 1991).

Todo ello podría hacer pensar que la comprensión ausubeliana es condición necesaria pero no suficiente para que se dé una adquisión duradera.

Si el objetivo de enseñanza es que el alumno ljege a una comprensión significativa de un determinado contenido, los mecanismos cognoscitivos propuestos por Ausubel parecen ser más que suficientes para conseguir dichos fines, y la información específica que se requiere del alumno es aquélla relacionada con los conceptos que van a ser enseñados.

Ahora bien, al ser dicha información:

- declarativa (dice qué se piensa sobre el contenido y no los mecanismos por los que se alcanza dicho pensamiento);
- estática (no informa sobre el grado de estabilidad de la idea);

- Limitada (no informa del grado de relación de la idea con el resto del bagaje cognoscitivo del alumno);

resulta insuficiente, creemos, si se busca algo más que la comprensión de lo enseñando.

\section{Según la propuesta piagetiana}

Si se pretende una enseñanza que lieve al alumno a utilizar los contenidos académicos como esquemas asi. miladores semejantes a los que se derivan de su interacción con el medio, se exige, considerando una propuesta neopiagetiana donde sc enfatiza los esquemas especifficos (Marín, 1995, pp 75-80):

- información específica relativa al esquema cognoscitivo utifizado para asimitar un determinado contenido concreto;

- información de indole general relacionada con los mecanismos que se ponen en juego en el mismo proceso de asimilación.

Esta última información debe estar ligada al nivel cog. noscitivo del alumno, pues dicho nivel se caracteriza por las capacidades y limitaciones del proceso asimilador de la estructura cognoscitiva.

La idea de considerar como información significativa los mecanismos de asimilación está notablemente influenciada por la epistemología piagctiana, pero, desde otras perspectivas independientes a la anterior, se ha propuesto considerar como información relevante Ias restricciones cognoscitivas de la actividad asimiladora del sujeto, que limitan y canalizan su capacidad interpretadora, constituyendo un marco de referencia que restringe los posibles tratamientos de los datos perceptivos y además son comunes a todos los individuos (Sebastia, 1992).

Llámense reglas de asimilación o restricciones cognoscitivas, bajo distinta terminología, se está hablando de lo mismo: sólo es posible adquirir un nuevo contenido si éste es asimilado a uno o varios esquemas ya integrados en la estructura cognoscitiva del sujeto (Pozo et al., 1991), y lo que al final se inserta en dicha estructura no será el nuevo contenido sino un «producto» filtrado por las limitaciones y capacidades en el proceso de asimilación de los esquemas utilizados.

Si la cuestión crucial a considerar en la enseñanza no es tanto que los alumnos entiendan los conceptos académi. $\cos$, sino que puedan utilizarlos y activarlos adecuadamente para interpretar los hechos a los que se enfrentan (Driver, 1988), parece que desde este y otros trabajos se está señalando la insuficiencia de que la información relevante del alumno para promover el aprendizaje significativo sea exclusivamente conceptual (Pozo, 1987; Sebastia, 1992). 


\section{IMPILICACIONES DIDÁC'TICAS AL DIFE- RENCIAR COMPRENSION Y ASIMILACION}

Entre comprender y asimilar existen diferencias que crecmos importante resaltar por lo que puede aportar a una mejor comprensión del aprendizaje de las ciencias. Conscientes de la complejidad de la cuestión, más que resolver la cuestión en el plano conceptual, se intentará hacerlo de un modo operativo.

Comprender es relacionar lo nuevo con lo ya existente. Comprender sin asimilar permite al sujeto poder expresar el mensaje «con otras palabras», pero nunca poder utilizarlo para resolver un problema nuevo o inferir algún tipo de conducta intelectiva frente al medio. En un entorno de enseñanza, el mayor alcance de la comprensión «académica» puede ser una exposición satisfactoria en un examen.

l.o comprendido sin proceso de asimilación es susceptible de olvido, por lo que parece plausible suponer que el proceso de asociación a un significado se da en una zona diferente a la MLP: la memoria de trabajo (MT) (Mathieu y Thomas, 1985, p. 241).

La comprensión viene cuando el sujeto vincula el significante externo con el significado que él aporta. Así, puede observarse desde distintas perspectivas:

- Psicológica. Existe consenso en admitir que es imposible comprender si no existe un elemento de la estructura cognoscitiva que aporte un significado (Pozo et al., 1991 ; Mayer, 1986); es necesaria la presencia de uno o varios esquemas en la memoria de trabajo para que se dé el proceso de comprensión.

- Epistcmológica. Se admite que el significado no está asociado al dato o información que se percibe; ésta es una actividad mental del sujeto, de tal sucrte que sujetos que han activado para ello esquemas diferentes dan significados diferentes.

Creemos plantear el problema en sus tírminos justos cuando se señala que las condiciones de aprendizaje, para que lo comprendido en la MT pase a la MLP, son más complejas que las que se requieren para llevar a cabo la comprensión, hasta el punto que esto último hay que verlo como requisito de lo primero.

Por ejemplo, el alumno puede comprender la información verbal contenida en el primer principio de la dinámica porque:

a) la naturaleza del material que se va a aprender ticne un significado lógico para el que lo aprende (si no hay nada que retenga al móvil, parece lógico que éste siga su marcha indefinidamente);

b) Jo puede vincular a contenidos cognoscitivos que existen en la cstructura cognoscitiva del sujeto (se puede apreciar en observaciones cotidianas que, en superficies más lisas, el movil mantiene por más tiempo su impulso inicial);
Gracias a esta adquisición, ante preguntas como:

- ¿Puede existir movimiento si no existc una fuer $\measuredangle$ a?

- ¿Qué se requiere para parar un móvil que se desiza por una superficie sin rozamiento?

las respuestas son satisfactorias.

Pero si se plantean cuestiones tales como:

- ¿Hasta dónde llegará una moneda que soplamos ligeramente si está en una superficie sin rozamiento?

- ¿Cómo puede salir una persona del centro de un lago helado si la superficie no presenta rozamicnto?

- ¿Por qué derrapa un coche?

entonces, las respuestas son más imprecisas.

$\mathrm{Y}$ ante la demanda de previsiones:

- ¿Cuánto más avanzará una moneda si echamos cera sobre la superficie?

- ¿Derrapará la bici al tomar aquella curva?

posiblemente las imprecisiones sean aún mayores.

Las condiciones de aprendizaje que se requieren para responder a todas las cuestiones de un modo más o menos satisfactorio deben contener la información comprendida e integrarla en la estructura cognoscitiva, mediante la participación de uno o varios esquemas ya existentes por procesos de asimilación y acomodación (Piaget, 1977a).

De este modo, el conocimiento integrado en un esquema:

- se hace operativo y sc puede aplicar en contextos diferentes;

- podría formar un nuevo esquema por diferenciación (Piaget y García, 1973);

- es susceptibie de extender su capacidad asimiladora mediante nuevos procesos de asimilación y acomodacion ante lo adquirido por nuevas interacciones del sujeto;

El paso de comprender a asimilar no es fácil y conlleva un alto coste en recursos didácticos y en tiempo de aprendizaje (habría que cuestionarse su rentabilidad didáctica), pues requiere del sujeto además de la comprensión:

- coherencia en el nuevo conocimiento;

- apreciar que el nuevo conocimiento responde satisfactoriamente ante las demandas que le plantea su entorno;

- que el medio le plantee problemas para cuya solución sea necesario o más conveniente utilizar cl nuevo conocimiento. 
en la formación de concepciones sobre diversos dominios de enseñanza.

d) Una técnica que permite recoger información tanto específica como general es la entrevista individual.

Las prucbas de papel y lápiz excesivamente mediatizadas por la estructura del propio contenido que se investiga, como se ha apreciado anteriormente, ofrecen sólo una primera toma de contacto con to que realmente son las concepciones de los atumnos.

Este modo de obtener los datos del alumnado podría llevar al investigador a concluir que las «concepciones» por él «descubiertas» no evolucionan o son difíciles de mođificar con el aprendizaje, pero también cabe la posibilidad de que, utilizando inferencias correctas, haya partido de premisas falsas.

Admitamos que no todo lo que catalogamos como «concepción» del alumno, realmente to sea, lo cual puede ocurrir si se utiliza una metodología que sesgue y distorsione demasiado los datos del alumno.

I a entrevista individual permite analizar cómo se acomodan los esquemas cognoscitivos específicos del alumno a variaciones de los distintos factores relacionados con la tarea. En primer lugar, permite hacer un análisis de las respuestas a nivel de significados y no de significantes, que, aunque supongan un problema también didáctico, no parecen ofrecer la misma resistencia. Además, obligando al esquema explicativo del alumno a aplicarlo ante una diversidad de situaciones físicas, se puede observar la estabilidad de dicho esquema y su capacidad de acomodarse a las peculiaridades de cada situación, lo que aporta una información sobre la capacidad de asimilación del esquema cognoscitivo que está utilizando.

e) Scgún se deduce de los dos apartados anteriores, el estudio de las concepciones debe ser doblemente evolutivo. Por un lado, habría que conocer cómo se va modificando un determinado esquema con la variabilidad de los distintos factores que intervienen en una situación y con las distintas situaciones y, por otro, cómo se va desarrollando en sujetos de diferente edad.

Como se puede apreciar, estas orientaciones metodológicas están deducidas principalmente de partes bien fundamentadas del entramado conceptual piagetiano (mecanismos de asimilación y acomodación, equilibración cognoscitiva, esquema, toma de conciencia, estrategias seguidas por el mismo Piaget para realizar sus entrevistas clínicas, etc.) (Marín, 1995, pp. 99-14I).

\section{CONCLUSIONES A MODO DE SÍNTESIS}

Se ha defendido que los distintos modelos usualmente utilizados en enseñanza de las ciencias llevan implícitos diferentes objetivos de enseñanza. Así, el modelo de Ausubel se refiere a la comprensión significativa de] contenido, mientras que el de cambio conceptual y, de forma más explícita, el de Piaget, están formulados para un aprendizaje operativo de dicho contenido. Este aprendizajc operativo implica la comprensión y además el contraste de la utilidad y eficiencia de ese nuevo contenido. Por tanto, consideramos que la comprensión es condición necesaria pero no suficiente para la asimilación del contenido.

La tendencia actual de investigar las concepciones de los alumnos de un modo descriptivo ofrece una informacion específica y limitada referente al contenido a enscĩar. Dichas concepciones podrían ser suficientes para discñar procesos de enseñanza y aprendizaje que pretendan la comprensión del significado del contenido.

Si lo que se pretende es que el nuevo conocimiento sea utilizado por el alumno en contextos diferentes, quizá sc requiera otra metodología para investigar las concepciones de los alumnos. Esta nueva metodología debe proporcionar tanto información específica relativa al contenido a enseñar como información general relacionada con la estabilidad de los esquemas de asimilación puestos en juego por los alumnos.

Con la intención de sintetizar esta metodología, se sugiere, por último, ciertos aspectos de la misma que favoreccrían el alcance de una información de las concepciones del alumno más significativa y completa. I os aspectos apuntados son:

- Contextualizar teóricamente las investigaciones sobre concepciones respetando, a la ve $z$, los criterios metodológicos exigibles en toda investigación.

- Categorizar las respuestas del alumno desde lo que sabe y no desde su distancia al contenido académico.

- Tener en cuenta tanto los aspectos conceptuales como los procesuales de los esquemas cognoscitivos.

- Utilizar la técnica de la entrevista individual como medio más ióónco para conocer la estabilidad de los esquemas asimiladores.

- Realizar estudios de concepciones de forma evolutiva. Dicha evolución debería referirse tanto a los sujetos de diferente edad como a los diferentes contextos y factores que intervienen en los mismos.

\section{NOTA}

El presente trabajo ha sido financiado por la DGICXT como parte del proyecto PS93-0174 del Programa Sectorial de Promoción General del Conocimiento. 


\section{REFERENCIAS BIBLIOGRÁFICAS}

ABIMBOLA, I.O. (1988). The problem of terminology in the study of student conceptions in science. Science Education, $72(2)$, pp. 175-184.

ACEVEDO, J.A. (1990). Aportaciones acerca del aprendizaje por analogía: nodelos analógicos y conceptuales de la corriente eléctrica. Cambio educativo y desarrollo profesional. Actas del VII Jornadas de Estudio sobre la Investigación en la Escuela, Sevilla, pp. 201-208.

ALIBERAS, I., GUTIÉRREZ, R. e IZQUIERDO, M. (1989). Modelos de aprendizaje en la didáctica de las ciencias. Investigación en la Escuela, 9, pp. 17-24.

AUSUBFL, D.P. (1982). Psicología educativa. Un punto de vista cognoscitivo. México: Trillas.

AUSUBEL, D.P., NOVAK, J.D. y HANESIAN, H. (1986). Psicología educativa. Un punto de vista cognoscitivo. México: Trillas.

BELTRÁN, J. (1993). Procesos, estrategias y técnicas de aprendizaje. Madrid: Síntesis.

BUNGE, M. (1981). La investigación cientifica. Barcelona: Ariel.

CARMICHAEL, P, et al. (1990). Rechearh on students' conceptions in science: a bibliography. University of Leeds: Children's learning in Science.

CARRETERO, M., LÓPEZ MANJÓN, A., POZO, J.L., LEÓN, J.A., PÉREZ ECHEVARRIA, P. y ASENSIO, M. (1992). Psicología de la instrucción, razonamiento y conocimientos especifficos. Infancia y Aprendizaje, 59, pp. 11-29.

CLAXTON, G. (1986). The altemative conceiver's conceptions. Studies in Science Education, 13, pp. 123-130.

CONFREY, J. (1990). A Review of the Research on Student Conceptions in Mathematics. Science and Programming, pp. 3-56, en Cazden, C. (eds.). American Education Research Association. Michigan State University. Review of Educational Research.

DRIVER, R. (1986). Psicología cognoscitiva y esquemas conceptuales de los alumnos. Enseñanza de las Ciencias, 4(1), pp. 3-15.

DRIVER, R. (1988). Un enfoque constructivista para el desarrollo del currículo en ciencias. Enseñanza de las Ciencias, 2(6), pp. 109-120.

DUSCHL, R.A. y GITOMER, D.H. (1991). Epistemological Perspectives on Conceptual Change: Implications for Educational Practice. Journal of Research in Science Teaching. 28(9), pp. 839-858.

GILBERT, J.K. y SWIFT, D.J. (1985). Towards a Iakatosian analysis of the piagetian and alternative conceptions research programs. Science Education, 69(5), pp. 681-696.

GÓMEZ, M.A., POZO, J.I., SANZ, A. y LIMÓN, M. (1992). La estructura de los conocimientos previos en química: una propuesta de núcleos conceptuales. Investigación en la Escuela, 18 , pp. 23-40.

HASHWEH, M.Z. (1988). Descriptive studies of students' conceptions in science. Journal of Research in Science Teaching, 25(2), pp. 121-134.

HERRON, J.D. (1978). Role of learning and development: Critique of Novak's Comparison of Ausubel and Piaget. Science Education, 62(4), pp. 593-605.
HEWSON, P.W. (1981). A conceptual change approach to learning science. European Journal of Science Edacation, 3, pp. 383-396.

HEWSON, P.W. y THORLEY, R. (I989). The conditions of conceptual change in the classroom. International Journal of Science Education, $11(5)$, pp. 541-553.

HIERREZUELO, J, y MONTERO, A. (1991). La ciencia de los alumnos. Su utilización en la didáctica de la física y química. Elzevir: Vélez Málaga.

JIMÉNEZ GÓMEZ, E., SOLANO, I. y MARÍN, N. (I994). Problemas de terminología en estudios realizados sobre «lo que el alumno saben en ciencias. Enseñanza de las Ciencias, 12(2), pp. 235-245.

LAWSON, A.E. (1983). Predicting Science achievement: the role of development level, disembedding ability, mental capacity, prior knowledge and beliefs. Joumal of Research in Science Teaching, 20(2), pp. 117-129.

LAWSON, A.E., BAKER, W.P., DIDONATO, L., VERDI, M.P. y JOHNSON, M.A. (1993). The role of hypotheticdeductive reasoning and physical analogues of molecular interactions in conceptual change. Journal of Research in Science Teaching, 30(9), pp. 1073-1085.

MARTÍNEZTORREGROSA, J., DOMÉNECH, J.J.y VERDÚ, R. (1993). Del derribo de ideas al levantamiento de puentes: "La epistemología de la ciencia como criterio organizador de la enseñanza de las ciencias fística y química». Qurriculum, 6, pp. 67-89.

MARÍN, N. (1992). La devaluación de las nociones previas en la teoría piagetiana. Investigación en la Escuela, 16, pp. 23-35.

MARÍN, N. (1994). Elementos cognoscitivos dependientes del contenido. Revista interuniversitaria de formación del profesorado, 20, pp. 195-208.

MARÍN, N. (1995). Metodología para obtener información del alumno de interés didáctico. Almería: ServiciodePublicaciones de la Universidad de Almería.

MARÍN, N. y BENARROCH, A. (1994). A comparative study of Piagetian and constructivist work on conceptions in science. lnt. J. Sci. Educ., 16(1), pp. 1-15.

MARÍN, N, y JIMÉNEZ GÓMEZ, E. (1992). Problemas metodológicos en el tratamiento de las concepciones de los alumnos en el contexto de la filosofía e historia de la ciencia. Enseñanza de las Ciencias, 10(3), pp. 335-339.

MATHIEU, J., y THOMAS, R. (1985). Manuel de Psychologie. París: Vigot.

MAYER, R.E. (1986). Pensamiento, resolución de problemas y cognición. Barcelona: Paidós.

MILLAR, R. (1989). Constructive criticisms. Int. J. Sci. Educ., 11, pp. 587-596.

MONK, M. (1990). A Genetic Epistemological Analysis of Data on Children's ideas about DC electrical circuits. Research in Science \& Technological Education, 8(2), pp. 133-143.

MOREIRA, M.A. (1994). Diez años de la revista Enseñanza de las Ciencias: de una ilusión a una realidad. Enseñanza de las Ciencias, 12(2), pp. 147-153.

NIAZ, M. (I99I). Role of the Epistemic Subject in Piaget's Genetic Epistemology and its importance for Science Education. 
Journal of Research in Science Teaching, 28(7), pp. $569-580$.

NOVAK, J.D. (1982). Teoria y práctica de la educación. Madrid: Alianza Universitaria.

NOVAK, J.D. y GOWIN, D.B. (1988). Aprendiendo a aprender. Barcelona: Martínez Roca. (Traducido de Learning how to learn. 1984, Cambridge University Press.).

PIAGLT, J. (1974). El estructtralismo. Barcelona: Oikos-Tau.

PLAGET, J. (1977a). Epistemología genética. Argentina: Solpin. (Traducido de L'epistemologie génetique. 1970. París: Presses Universitaires de France.)

PIAGHE, J. (1977b). Lógica y psicología. Argentina: Solpin. (Traducido de Logic and psychology. 1953. Nueva York: Manchester University Press.)

PIAGET, J. (1978). La equilibración de las estructuras cognitivas, Problema central del desarrollo. Vol. a. Madrid: Siglo XXI.

PIAGET, I. (1980). Psicologia y pedagogia. Barcelona: Ariel. (Traducido de Psychologie et pédagogie. 1969. París: Gonthier.)

PIAGET, J. (1984). Psicología de la inteligencia. Buenos Aires: Psique. (Traducido de La psychologie de lintelligence. 1947. París: A. Colin.)

PIAGFT, J. c INHELDER, B. (1948). La représentation de l'espace chez l'enfant. París: PUF.

PIAGET, J. e INHELDER, B. (1971). El desarrollo de las cantidades en el niño. Barcelona: Nova Terra. (Traducido de Le développement des quantités physiques. 1941 . París: Delachaux \& Niestle.)

PIAGHT, J. y GARCÍA, R. (1973), Las explicaciones causales. Barcelona: Barral.

POSNER, G.J., STRIKF, K.A., HEWSON, P.W. y GERTZOG, W.A. (1982). Accomodation of a Scientific Conception: Toward a Theory of conceptual change. Science Education, 66(2), pp. 211-227.

PO7.O, J.I. (1987). Aprendizaje de la ciencia y pensamiento catusal. Madrid: Visor.

POZO, J.I., GÓMEZ CRESPO, M.A., LIMÓN, M. Y SERRANO SAN7, A. (1991). Procesos cognitivos en la comprensión de las ciencias: las ideas de los adolescentes sobre la química. Madrid: CIDE (MEC).
RIVIFRF, A. (1992). Sobre psicología de la instrucción, razonamiento y conocimientos específicos, Comentarios a la ponencia de $\mathrm{M}$. Carretero y otros. Infancia y aprendizaje, 59 , pp. 31-33.

ROTH, W.M. (1990). Neo-piagetian predictors of achievement in physical science. Journal of Research in Science Teaching, $27(6)$, pp. 509-521.

SEBASTIA, J.M. (1992). Constructivismo restringido: i una perspectiva viable? International Conference of History of the Physical-Mathematical Sciences and the Teaching of Sciences, Madrid, pp. 160-161.

SEBASTIA, J.M. (I993). ¿Cuál brilla más?: Predicciones y reflexiones acerca del brillo de las bombillas. Enseñanza de las Ciencias, $11(1)$, pp. 45-50.

SERRANO,J.A. (1978). Pensamiento y concepto. México:Trillas.

SHAYER, M. y ADEY, L'. (1984). La ciencia de enseñar ciencia. Desarrollo cognoscitivo y exigencias del currículo. Madrid: Narcea.

SHAYER, M. Y ADEY, P.S. (1992a). Accelerating the development of formal thinking in middle and high school students II: Postproject effects on Science achicvement. Journal of Research in Science Teaching, 29(1), pp. 81-92.

SHAYER, M. Y ADEY, P.S. (1992b). Accelerating the development of formal thinking in the middle and high school students III: Testing the permanence of effects. Journal of Research in Science Teaching, 29(10), pp. 1101 1115 .

SHAYER, M. y ADEY,P.S (1993), Accelerating the development of formal thinking in middole and high school students IV: three years after a two year intervention. Journal of Research in Science Teaching, 30 (4), pp. 351-366

STAVY, R. (1990). Pupils' problems in understanding conservation of matter. Internacional Journal of Science Education, 12(3), pp. 50l-5!2

STRIKE, K.A. y POSNER, G.J. (1990). A revisionist theory of conceptual change, en Duschl, R, y Hamilton, R. (eds.). Philosophy of Science, Cognitive Science and Educacional Theory and Practice. Nucva York: Suny Press.

VILLANI, A. y PACCA, J.L.A. (1990). Conceptos espontáneos sobre colisiones. Enseñanza de las Ciencias, $8(3)$, pp. 238-243.

VUYK, R. (1985). Panoramica y crítica de la epistemología genética de Piaget 1965-1980. Madrid: Alianza Universitaria.

[Artículo recibido en enero de 1996 y aceptado en noviembre de 1996.] 